\title{
Thibault LE TEXIER, Le Maniement des hommes. Essai sur la rationalité managériale
}

Lucas Manerville

\section{OpenEdition}

1 Journals

Édition électronique

URL : http://journals.openedition.org/ress/3569

DOI : $10.4000 /$ ress.3569

ISBN : $1663-4446$

ISSN : 1663-4446

Éditeur

Librairie Droz

Édition imprimée

Date de publication : 30 novembre 2016

Pagination : 295-298

ISSN : 0048-8046

Référence électronique

Lucas Manerville, "Thibault LE TEXIER, Le Maniement des hommes. Essai sur la rationalité managériale 》, Revue européenne des sciences sociales [En ligne], 54-2 | 2016, mis en ligne le 18 août 2016, consulté le 25 septembre 2020. URL : http://journals.openedition.org/ress/3569; DOI : https://doi.org/10.4000/ ress.3569

Ce document a été généré automatiquement le 25 septembre 2020.

(C) Librairie Droz 


\title{
Thibault LE TEXIER, Le Maniement des hommes. Essai sur la rationalité managériale
}

\author{
Lucas Manerville
}

\section{RÉFÉRENCE}

Thibault LE TEXIER, 2016, Le Maniement des hommes. Essai sur la rationalité managériale, Paris, La Découverte, 288 p.

1 C'est une histoire du management par les textes que nous propose Thibault Le Texier dans son ouvrage. Commencée au début du XVIII ${ }^{\mathrm{e}}$ siècle, elle se poursuit jusqu'à nos jours, au gré des modifications sémantiques du terme, de l'amélioration des techniques de gestion, de la pénétration toujours plus profonde de nos sociétés par cette rationalité nouvelle. L'auteur distingue trois moments: si l'usage du mot «management» est d'abord confiné à la sphère domestique, des mécaniciens américains l'en extraient dans les années 1830 et l'emploient pour désigner le soin et la gestion des machines. À la fin du XIx ${ }^{\mathrm{e}}$, le terme s'applique au management des hommes, bouclant le glissement sémantique d'une "praxis du soin » à une "science du contrôle » (p.13). L'ouvrage s'applique à analyser les conditions, les caractéristiques et les conséquences de ce glissement, en trois chapitres de longueurs équivalentes.

2 Le premier chapitre analyse les conditions d'évolution du concept de management. Articulé autour de quatre retournements de valeurs constatés entre la fin du XIX et le début $\mathrm{du} \mathrm{xx}^{\mathrm{e}}$ siècle, il montre comment s'est formé le terreau propice à l'épanouissement du management dans son sens contemporain. L'auteur souligne d'abord que le XIX ${ }^{\mathrm{e}}$ siècle voit s'imposer l'efficacité comme valeur-étalon fondamentale. Il détecte dans la littérature de l'époque le glissement d'un idéal d'individu industrieux à celui d'individu efficace. Le premier management exhorte les êtres humains à ne pas rester oisifs, le travail représentant une fin en soi. Au tournant du siècle, en revanche, 
c'est le résultat du travail accompli qui retient l'attention, ouvrant ainsi la voie à la sujétion de l'individu à un output extrinsèque à son savoir-faire. Les deux glissements suivants (de l'arrangement à l'organisation de l'espace d'une part, du calcul à la rationalisation de l'autre) reflètent le souci toujours croissant de façonner les hommes. Le premier management ne voyait dans la collecte de données qu'un moyen de décrire l'environnement et de l'adapter à l'individu. Peu à peu émerge l'idée que l'être humain aussi doit se plier aux exigences de l'efficacité. Il ne s'agit dès lors plus, par exemple, d'adapter la chaîne de production aux lois de l'ergonomie, mais de concevoir l'agencement de l'usine comme un outil contraignant l'ouvrier à améliorer sa productivité. Le dernier glissement est celui du soin au contrôle. On le conçoit aisément en considérant le changement d'objet du management: plantes, animaux, enfants, malades dans un premier temps, travailleurs et cadres à partir $d u x^{e}$ siècle. De ces quatre glissements Le Texier déduit trois thèses. D'abord, les conséquences de l'essor de la pensée managériale outrepassent largement les limites de la sphère économique. Ensuite, la pensée managériale désindividualise l'humain et le façonne rationnellement en fonction d'un but à atteindre - l'augmentation de l'efficacité. Enfin, le management cherche à substituer aux tendances «naturelles » de l'être humain les prescriptions et les normes issues de ses rationalisations.

Dans un deuxième chapitre, l'auteur s'intéresse aux singularités du management moderne. Le management n'est ni une science ni une idéologie. C'est une discipline avant tout pragmatique, dépourvue d'un corpus détaillé de connaissances théoriques. Il s'agit en fait d'une façon empirique de concevoir et modeler le monde à partir de quelques grands principes structurants. C'est en ce sens que l'auteur parle de « rationalité managériale ». Cette rationalité managériale, tout d'abord cantonnée au monde du travail, va peu à peu envahir le champ de la consommation, profitant une nouvelle fois des limitations méthodologiques d'une science économique manquant de pragmatisme. Commencé par la standardisation des produits, qui marginalise les ressorts traditionnels de la vente (négociation, importance des relations interpersonnelles), le mouvement se poursuit via le conditionnement du consommateur, dont on cherche à prévoir et orienter les choix en s'appuyant sur un contrôle et une participation accrus. "Chargé d'un nombre croissant de tâches de production (imprimer ses billets de train, par exemple), de mesure (évaluer des produits ou des salariés), de contrôle (passer sa carte de transport sur des bornes) ou encore de surveillance (signaler des bagages abandonnés), le consommateur incorpore toujours plus de réflexes standardisés, d'éléments de langage et de protocoles opératoires. Il voit managés ses besoins, ses souhaits, ses gestes, ses habitudes, ses opinions, ses connaissances et ses sentiments. Comme n'importe quel employé, il peut être stimulé, motivé, formé » (p. 143). En substance, ce que cherche in fine la rationalité managériale, ce n'est pas le profit maximal, mais l'efficacité maximale. C'est en cela que la rationalité managériale s'oppose le plus explicitement à la rationalité marchande. Il ne s'agit pas de dégager la marge la plus importante, mais d'offrir les outputs les plus utiles, produits selon les méthodes les plus efficaces. Ce chapitre se conclut par ce constat que la rationalité managériale recouvre aujourd'hui des pans entiers de notre existence, sphère privée, travail, consommation.

4 Le troisième et dernier chapitre traite du pouvoir managérial et de ses institutions. Le Texier souligne que les principes du premier management sont largement influencés par le milieu de leur genèse : entreprises ferroviaires et armée. En outre, l'exploitation 
agricole et le foyer élargi qui en assure la cohérence restent, tout au long du XIX siècle, les formes d'organisation sociale dominantes, marquées par la prééminence des relations patriarcales. Ces principes sont cependant énergiquement remis en cause par la classe naissante des managers. Générateurs d'interactions personnalisées, de principes fluctuants et tacites, d'incertitudes et d'inexactitudes, ils sont peu à peu remplacés par des règles formelles et uniformes, dont l'objectif est d'atteindre un rendement maximal et mesurable. Il ne faut pas comprendre cette exigence de rendement comme un calcul à courte vue sur les performances des employés au jour le jour, mais comme une façon nouvelle d'envisager les rapports entre employés et direction dans l'entreprise. Les managers scientifiques réclament ainsi une amélioration de la salubrité des usines, un soin accru apporté à la santé des ouvriers. Mais ces exhortations, loin de trouver leur origine dans une philanthropie teintée de paternalisme, ont en réalité pour but avoué d'augmenter la productivité des employés. Comme le rappelle l'auteur, «ni le bien-être, ni la santé, ni le soin ne sont les fins en soi de cette démarche ; son but, c'est l'efficacité » (p. 199). Le déclin du patriarcat permet d'engager une universalisation du management, au moins dans les discours. Ainsi de l'État qui se conçoit toujours plus comme une immense entreprise. À l'autre bout du spectre, les principes du management s'érigent en balises pertinentes pour conduire sa vie privée. Toute une littérature exhorte à reconsidérer les relations sociales ou familiales, les habitudes les plus élémentaires, les choix cruciaux, les tâches les plus insignifiantes, sous le prisme de l'efficacité, de la gestion de projet. C'est un «nouveau sens commun» (p. 241) qui se fait jour, technique, sans épaisseur ni transcendance, dans lequel on fait tenir l'individu, l'enserrant avec bienveillance dans un moule qu'il sera le premier à vouloir épouser.

5 La conclusion, qui suit immédiatement cet alarmant constat, s'attache d'abord à définir ce qu'est une institution, combinaison d'un "groupe humain", de "procédures", d'« agencements instrumentaux » et d'une « rationalité » (p. 246) dans le sens que lui a donné l'auteur plus haut dans l'ouvrage. Le visage du pouvoir au sein des institutions dépend des rationalités qui les dominent. L'auteur critique Foucault et Bourdieu pour ne pas avoir été assez radicaux dans leur remise en cause de la notion de pouvoir, pour avoir finalement renoncé à le décentraliser tout à fait, revenant pour l'un à la prégnance du droit, pour l'autre à celle de l'État.

6 L'ouvrage, extrêmement documenté, nous convie à un passionnant voyage dans l'histoire du management. La notion de rationalité, sorte d'épistémè à taille humaine, en tout cas sociale et organisationnelle, nous semble fort bien adaptée à l'analyse du phénomène, son émergence, ses transformations. L'appel à une nouvelle théorie du pouvoir convainc naturellement. Nous serons en revanche plus réservés quant au parti pris résolument anti-managérial qu'affiche l'auteur. On ne peut qu'être surpris par la soudaine comparaison du management et de l'eugénisme dans le cours du deuxième chapitre. Car si la fin de l'eugénisme est l'efficacité, si pour y parvenir les eugénistes s'appuient sur la collecte des données, Le Texier nous rappelle ensuite, convoquant les travaux du médecin physiologiste Jules Amar et de l'entrepreneur et philanthrope Ernest Solvay, que l'eugéniste cherche à provoquer l'« avènement d'un type d'homme supérieur» (p.119-120), et conçoit l'efficacité comme «le but suprême éminemment moral à poursuivre » (p.118-119, souligné par nous). Deux caractéristiques essentielles totalement étrangères à la pensée managériale. Ce que révèle cette comparaison outrée, c'est l'idée centrale de l'ouvrage selon laquelle l'efficacité est une valeur, au même titre que la justice, l'honneur ou le respect de la tradition. Or il nous semble qu'il 
y a une profonde différence de nature entre cette notion-là et ces notions-ci. Car l'honneur, la tradition ou la justice obéissent à des règles a priori et intrinsèques, tandis que l'efficacité est toute tournée vers un output. L'efficacité peut ainsi, à l'exclusion des autres notions considérées, s'appliquer sans mal à des activités non humaines. L'efficacité est mesurée dans le résultat d'une action, qu'elle soit ou non déterminée par un choix moral. Fondamentalement amorale, elle ne peut qu'être dépourvue de valeur. Ainsi donc, si le postulat de l'efficacité est dérangeant, il l'est précisément au nom de certaines valeurs. Il faudrait au contraire reconnaître à l'efficacité un statut à part, qui la préviendrait contre toute tentative de comparaison avec une quelconque valeur morale, et ouvrirait la voie à un questionnement de la notion, à une tentative de définition. On éviterait ainsi la vision apocalyptique de l'homme contemporain qui précède immédiatement la conclusion, vision qui, sans doute, pèche par idéalisation du passé. Car quelle époque de l'humanité peut se targuer d'avoir amené la plupart des individus de ses populations à "être les créateurs nietzschéens de [leur] destin » (p. 243)?

\section{AUTEURS}

\section{LUCAS MANERVILLE}

Tokyo, Société Michelin 